\title{
ÜBER AUTOMORPHIEFAKTOREN UND DIE DEDEKINDSCHEN SUMMEN
}

\author{
von ARMIN LEUTBECHER
}

(Received 15 November, 1968)

Einleitung. Bei der Betrachtung automorpher Formen zu diskreten Untergruppen $\Gamma$ von $S L_{2}(\mathbf{R})$ mit nicht ganzzahligem Gewicht bzgl. des Automorphiefaktors $J(A, z)=$ $(c z+d)^{-2}, A=\left(\begin{array}{ll}a & b \\ c & d\end{array}\right)$, hat man einen $Z$ weig $L(A, z)$ des Logarithmus von $J(A, z)$ zu fixieren. Durch

$$
2 \pi i W(A, B)=L(A B, z)-L(A, B z)-L(B, z)
$$

wird dann ein 2-Kozyklus $W$ von $S L_{2}(\mathbf{R})$ mit Koeffizienten in $\mathbf{Z}$ definiert. Die verschiedenen Zweige von $\log J(A, z)$ liefern gerade die sämtlichen Kozyklen einer Kohomologieklasse. Indes existieren Fixierungen von $L(A, z)$, für die $W$ symmetrisch, d.h. stets $W(A, B)=W(B, A)$, ist. Das folgt aus einem allgemeinen Kriterium in Lemma 1. Die explizite Konstruktion von $L(A, z)$ mit symmetrischem $W$ bringt Abschnitt 2. Anwendungen auf automorphe Formen werden im dritten Abschnitt gegeben. Insbesondere ergibt sich eine einigermaßen handliche Form für die "Periode"

$$
\omega(\eta, A)=\frac{1}{2 \pi i}\left\{\int_{z}^{A z} \frac{\eta^{\prime}(t)}{\eta(t)} d t+\frac{1}{4} L(A, z)\right\}, \quad A \in S L_{2}(\mathbf{Z})
$$

der Dedekindschen Funktion

$$
\eta(z)=e^{2 \pi i z / 24} \prod_{m=1}^{\infty}\left(1-e^{2 \pi i m z}\right)
$$

Den wesentlichen Bestandteil in $\omega(\eta, A)$ bilden bekanntlich die Dedekindschen Summen $s(d, c)$. Da $W$ symmetrisch gewählt wurde, wird $\omega(\eta, A)$ eine Klassenfunktion auf $S L_{2}(\mathrm{Z})$. Daraus folgen die meisten der bekannten Identitäten für die Dedekindschen Summen.

Die Existenz einer Abbildung $\omega: \Gamma \rightarrow C$ mit der Eigenschaft

$$
\omega(A B)=\omega(A)+\omega(B)+W(A, B),
$$

wie sie durch $\omega(A)=4 \omega(\eta, A)$ gegeben wird-bedeutet nichts anderes als das Zerfallen einer durch $W$ definierten zentralen Gruppenerweiterung. Daß sie im Falle $\Gamma=S L_{2}(\mathrm{Z})$ zerfällt, ist eine Konsequenz elementarer Sätze der Kohomologietheorie. Weil $\Gamma /\left\{ \pm\left(\begin{array}{ll}1 & 0 \\ 0 & 1\end{array}\right)\right\}$ freies Produkt von zyklischen Gruppen endlicher Ordnung ist, ist die Abbildung $\omega$ eindeutig bestimmt. Man hat dadurch eine von der Dedekindschen Funktion unabhängige Definition für $\omega$. 
Mit Hilfe der Dedekindschen Summen hat Wohlfahrt [10] eine Serie von Nichtkongruenzuntergruppen in $S L_{2}(\mathrm{Z})$ gefunden. In unserer Terminologie gesprochen betrachtet er für verschiedene Primzahlen $p, q$ den durch

$$
\chi(A)=\exp \left(\frac{12 \pi i}{q}\right)\left\{\omega\left(P A P^{-1}\right)-\omega(A)\right\}, \quad P=\left(\begin{array}{ll}
p & 0 \\
0 & 1
\end{array}\right)
$$

auf der Gruppe $\Gamma_{0}(p)$ der Matrizen $A=\left(\begin{array}{ll}a & b \\ c & d\end{array}\right) \in S L_{2}(Z)$ mit $c \equiv 0(\bmod p)$ definierten Charakter $\chi$ und zeigt, daß der Kern von $\chi$ eine Nichtkongruenzuntergruppe von $S L_{2}(Z)$ ist, wenn $q$ kein Teiler von $p^{2}-1$ ist. Dieses Resultat ergänzen wir im letzten Abschnitt durch die folgende Feststellung: Für natürliche Zahlen $n>1$ sei $N=\left(\begin{array}{ll}n & 0 \\ 0 & 1\end{array}\right), \Gamma_{0}(n)$ die Gruppe der $\left(\begin{array}{ll}a & b \\ c & d\end{array}\right) \in S L_{2}(\mathbf{Z})$ mit $c \equiv 0(\bmod n)$ und $\Omega_{n}(A)=6\left\{\omega\left(N A N^{-1}\right)-\omega(A)\right\} . \quad \Omega_{n}: \Gamma_{0}(n) \rightarrow \mathbf{Z}$ ist offenbar ein Homomorphismus, und es gilt: $\Omega_{n}^{-1}(m \mathbf{Z})$ ist dann und nur dann eine Kongruenzuntergruppe von $S L_{2}(Z)$, wenn $m$ ein Teiler von 24 ist.

1. Es sei $G$ eine Gruppe mit neutralem Element $I$ und $M$ eine (additiv geschriebene) abelsche Gruppe, aufgefaßt als trivialer $G$-Modul. $Z^{2}(G, M)=Z^{2}$ bezeichne die additive Gruppe der Abbildungen $w: G \times G \rightarrow M$, welche der Identität genügen

$$
w(A, B)+w(A B, C)=w(A, B C)+w(B, C) \text { für alle } A, B, C \in G
$$

ferner sei $B^{2}(G, M)=B^{2}$ die Untergruppe der $w \in Z^{2}$, für die mit einer passenden Abbildung $\alpha: G \rightarrow M$ gilt

$$
w(A, B)=\alpha(A)-\alpha(A B)+\alpha(B) .
$$

Die Faktorgruppe $Z^{2} / B^{2}$ ist bekanntlich isomorph zur zweiten Kohomologiegruppe $H^{2}(G, M)$ von $G$ mit Koeffizienten in $M$. Aus (1.1) kommt für alle $w \in Z^{2}$ und alle $A, B \in G$

$$
\left\{\begin{array}{l}
\text { (a) } w(A, I)=w(I, A)=w(I, I), \\
\text { (b) } w\left(A, A^{-1}\right)=w\left(A^{-1}, A\right), \\
\text { (c) } w\left(A B, B^{-1} A^{-1}\right)+w(A, B)+w\left(B^{-1}, A^{-1}\right)=w\left(A, A^{-1}\right)+w\left(B, B^{-1}\right)+w(I, I) .
\end{array}\right.
$$

Wir betrachten die Untergruppe $S$ von $Z^{2}$ der "symmetrischen" $w$ (d.h. $w(A, B)=w(B, A)$ für alle $A, B \in G)$, die von ihr erzeugte Untergruppe $\widetilde{S}=\left(S+B^{2}\right) / B^{2}$ von $H^{2}(G, M)$ und zeigen.

LEMMA 1. Für $w \in Z^{2}$ ist $\tilde{w}:=w+B^{2}$ in $\tilde{S}$ genau dann, wenn für alle vertauschbaren $A, B \in G$ gilt $w(A, B)=w(B, A)$.

Beweis. Die eine Richtung der Behauptung ist trivial. Ist nämlich $w_{0} \in S$ und mit einer Abbildung $\alpha: G \rightarrow M \quad w(A, B)=w_{0}(A, B)+\alpha(A)-\alpha(A B)+\alpha(B)$, so ist offenbar für je zwei vertauschbare $A, B \in G$ auch $w(A, B)=w(B, A)$. Zum Beweis der anderen Richtung machen wir eine Vorbemerkung. $H^{2}(G, M)$ ist bekanntlich isomorph zur Gruppe Opext $\left(M, G, \chi_{0}\right)$ 
der zentralen Gruppenerweiterungen von $M$ mit $G$ (vgl. MacLane [5, Chapter IV]). Ein Isomorphismus läßt sich folgendermaßen beschreiben: Ist

$$
E: 0 \rightarrow M_{\vec{q}} G \underset{p}{\rightarrow} G \rightarrow 1
$$

eine zentrale Gruppenerweiterung, also eine exakte Sequenz von Gruppenhomorphismen, für welche $q(M)$ im Zentrum von $\widetilde{G}$ liegt, so hat man zu jeder Abbildung $v: G \rightarrow \widetilde{G}$ mit der Eigenschaft $p \circ v=\operatorname{Id}_{G}$ (wir nennen $v$ dann ein Vertretersystem von $G$ in $\widetilde{G}$ für $E$ ) durch

$$
v(A) v(B) v(A B)^{-1}=q(w(A, B))(A, B \in G),
$$

ein $w \in Z^{2}$ definiert; und die verschiedenen Vertretersysteme $v^{t}$ von $G$ in $\widetilde{G}$ für $E$ liefern die sämtlichen $w^{\prime} \in w+B^{2}$. Ist andererseits $w \in Z^{2}$, so definiert

$$
(a, A)(b, B):=(a+b+w(A, B), A B) \text { für } a, b \in M \text { und } A, B \in G
$$

eine Gruppenstruktur auf $\widetilde{G}:=M \times G$ mit dem neutralen Element $(-w(I, I), I)$. Das durch $q(a):=(a-w(I, I), I), p(a, A)=A$ gegebene Paar von Abbildungen $q: M \rightarrow \widetilde{G}, p: \widetilde{G} \rightarrow G$ definiert eine zentrale Gruppenerweiterung $E: 0 \rightarrow M_{q} \underset{G_{p}}{G} G \rightarrow 1$, und $w$ gehört zu dem Vertretersystem $v(A)=(0, A)$. Dem Nullelement von $H^{2}(G, M)$ entspricht die Klasse der zerfallenden Erweiterungen, das sind diejenigen, für die ein Homomorphismus $v$ mit $p \circ v=\mathbf{I d}_{G}$ existiert.

Sei nun für ein $w \in Z^{2} E$ die so definierte Gruppenerweiterung und $v$ das durch $v(a)=(0, A)$ definierte Vertretersystem. Für den Kommutator zweier Elemente $g=(a, A), h=(b, B) \in \tilde{G}$ hat man

$$
\begin{aligned}
& g h g^{-1} h^{-1} \\
& \quad=\left(w(A, B)+w\left(A^{-1}, B^{-1}\right)+w\left(A B, A^{-1} B^{-1}\right)-w\left(A, A^{-1}\right)-w\left(B, B^{-1}\right)-2 w(I, I), A B A^{-1} B^{-1}\right) .
\end{aligned}
$$

Daraus liest man mit (1.2) ab: Ist für vertauschbare $A, B$ stets $w(A, B)=w(B, A)$, so gilt für $g, h \in \widetilde{G} g h g^{-1} h^{-1} \in q(M)$ nur, wenn $g h=h g$ ist. Anders ausgedrückt: Ist $p\left(g h g^{-1}\right)=p(h)$, so ist $g h g^{-1}=h$. Die Einschränkung von $p$ auf jede Klasse $K$ von in $H$ konjugierten Elementen ist dann also injektiv. Daraus folgt leicht, daß ein Vertretersystem $v_{0}$ von $G$ in $\tilde{G}$ existiert, für welches $v_{0}(G)$ aus vollen Klassen in $\widetilde{G}$ konjugierter Elemente besteht: Sei $\mathfrak{B}$ die Gesamtheit aller Teilmengen $V$ von $\widetilde{G}$ mit den beiden Eigenschaften

(1) Die Einschränkung von $p$ auf $V$ ist injektiv.

(2) $V$ besteht aus vollen Klassen konjugierter Elemente.

Nach dem Zornschen Lemma enthält $\mathfrak{B}$ ein bzgl. der Inklusion $\subset$ maximales Element $V_{0}$. Es ist $p\left(V_{0}\right)=G$. Sonst gibt es nämlich ein $h \in \widetilde{G}$ mit $p(h) \in G-p\left(V_{0}\right)$. Wegen (2) enthält dann $V_{0}$ kein Element der Klasse $K$ zu $h$ in $\widetilde{G}$ konjugierter Elemente, also ist $p(K) \cap p\left(V_{0}\right)=\emptyset$. Daraus folgt $K \cup V_{0} \in \mathfrak{B}$ im Widerspruch zur Maximalität von $V_{0}$. Also existiert ein Vertretersystem $v_{0}: G \rightarrow \widetilde{G}$, für das $v_{0}(G)=V_{0}$ aus vollen Klassen konjugierter Elemente besteht. Sei $w_{0}$ das $v_{0}$ vermöge

$$
v_{0}(A) v_{0}(B) v_{0}(A B)^{-1}=q\left(w_{0}(A, B)\right)
$$


zugeordnete Element von $Z^{2}$. Es ist $w+B^{2}=w_{0}+B^{2}$ und für alle $A, B \in G$ gilt

also

$$
\begin{aligned}
v_{0}(B A) & =v_{0}\left(A^{-1}\right) v_{0}(A B) v_{0}\left(A^{-1}\right)^{-1}, \\
q\left(w_{0}\left(B A^{A^{-1}}\right)\right) & =v_{0}(B A) v_{0}\left(A^{-1}\right) v_{0}(B)^{-1}
\end{aligned}
$$

$$
=v_{0}\left(A^{-1}\right) v_{0}(A B) v_{0}(B)^{-1}=q\left(w_{0}\left(A^{-1}, A B\right)\right) \text {. }
$$

Aus (1.2) kommt deshalb

$$
0=w_{0}\left(B A, A^{-1}\right)-w_{0}\left(A^{-1}, A B\right)=w_{0}(A, B)-w_{0}(B, A) .
$$

Das beendet den Beweis von Lemma 1.

Wir schließen diesen Abschnitt mit zwei Bemerkungen.

(1.3) Ist $w \in S$, so ist $w(A, B)=w\left(C A C^{-1}, C B C^{-1}\right)$ für alle $A, B, C \in G$.

(1.4) Ist für ein $w \in Z^{2}$ und gegebene $A, B \in G \quad w(A, X)=w(X, A)$ und $w(B, X)=w(X, B)$ für alle $X \in G$, so gilt auch $w(A B, Y)=w(Y, A B)$ für alle $Y \in G$.

Beweis. Aus (1.1) kommt mit der Voraussetzung von (1.3)

$$
\begin{aligned}
w\left(C \cdot A C^{-1}, C B C^{-1}\right) & =w\left(C, A B C^{-1}\right)+w\left(A C^{-1}, C \cdot B C^{-1}\right)-w\left(A C^{-1}, C\right) \\
& =w\left(A \cdot B C^{-1}, C\right)+w\left(A, B C^{-1}\right)-w\left(B C^{-1}, C\right)=w(A, B) .
\end{aligned}
$$

Entsprechend liefert die Voraussetzung von (1.4)

$$
\begin{aligned}
w(A . B, Y)-w(Y, A . B) & =w(A, B Y)+w(B, Y)-w(Y A, B)-w(Y, A) \\
& =w(B Y, A)+w(B, Y)-w(B, Y A)-w(Y, A)=0 .
\end{aligned}
$$

2. Sei für den Augenblick $X$ ein einfach zusammenhängendes Gebiet im $\mathbf{C}^{\mathbf{n}}$ und $G$ eine Gruppe mit neutralem Element 1 , welche als Gruppe analytischer Automorphismen auf $X$ operiert, ferner $J$ ein Automorphiefakter zu $(G, X)$, d.h. $J(A, z)$ ist für jedes $A \in G$ eine holomorphe Funktion auf $X$, und es gilt

$$
J(A B, z)=J(A, B z) J(B, z), \quad J(I, z)=1 .
$$

Dann ist $J(A, z)$ nullstellenfrei. Für jeden Zweig $L(A, z)$ des Logarithmus von $J(A, z)$ gilt

$$
L(A B, z)=L(A, B z)+L(B, z)+2 \pi i W(A, B), \quad A, B \in G,
$$

worin $W \in Z^{2}(G, \mathbf{Z})$ ist. Die verschiedenen Zweige $L^{\prime}$ des Logarithmus liefern offenbar die sämtlichen $W^{\prime}$ der Klasse $W+B^{2}(G, \mathbf{Z})$. Wir betrachten hier $G=S L_{2}(\mathbf{R})$ als Gruppe lineargebrochener Transformationen der oberen Halbebene $X=\mathfrak{H}=\{z \in \mathbf{C} ; \operatorname{Im} z>0\}$ und die beiden Automorphiefaktoren $j(A, z):=c z+d, J(A, z):=(c z+d)^{-2}$. Bei Petersson [7] wird ein Zweig $l_{1}(A, z)$ des Logarithmus von $j(A, z)$ fixiert durch

$$
-\pi<\operatorname{Im} l_{1}(A, z) \leqq \pi .
$$

$w_{1}$ sei der gemäß (2.1) zu $l_{1}$ gehörige 2-Kozyklus in $Z^{2}(G, Z)$. Für ihn gilt offenbar stets $\left|w_{1}(A, B)\right| \leqq 1$. Für vertauschbare $A, B \in S L_{2}(\mathbf{R})$ ist

$$
2 \pi i\left(w_{1}(A, B)-w_{1}(B, A)\right)=l_{1}(A, z)-l_{1}(A, B z)+l_{1}(B, A z)-l_{1}(B, z) .
$$


Die beiden Transformationen $z \rightarrow A z, z \rightarrow B z$ der Riemannsphäre $\overline{\mathbf{C}}$ besitzen einen gemeinsamen Fixpunkt $z_{0}$ in $\mathfrak{Y}$ oder $\operatorname{Rd} \mathfrak{S}$. Ist $z_{0} \in \mathfrak{S}$, so ergibt sich für $z=z_{0} \quad w_{1}(A, B)=w_{1}(B, A)$. Ist $z_{0} \in \operatorname{Rd} \mathfrak{G}$, so lassen sich $l_{1}(A, z)$ und $l_{1}(B, z)$ stetig nach $z_{0}$ fortsetzen, und wieder folgt $w_{1}(A, B)=w_{1}(B, A)$. Lemma 1 ergibt also, da $w_{1}+Z^{2}(G, \mathbf{Z})$ einen symmetrischen 2Kozyklus enthält. Allerdings folgt aus der Relation $(U T)^{3}=I$ für die Matrizen $U=\left(\begin{array}{ll}1 & 1 \\ 0 & 1\end{array}\right)$, $T=\left(\begin{array}{rr}0 & 1 \\ -1 & 0\end{array}\right), I=\left(\begin{array}{ll}1 & 0 \\ 0 & 1\end{array}\right) \in S L_{2}(\mathbf{R})$ leicht, daß jede Wahl von $l(A, z)=\log (c z+d)$, die außer von $z$ nur von der zweiten Zeile $(c, d)$ von $A$ abhängt, keinen symmetrischen Kozyklus liefert.

Für $A=\left(\begin{array}{ll}a & b \\ c & d\end{array}\right) \in S L_{2}(\mathbf{R})$ mit der Spur $\operatorname{tr} A=a+d$ definieren wir drei Funktionen $\beta, \delta, \varepsilon$ durch

$$
\begin{aligned}
& \beta(A)=\left\{\begin{array}{l}
1, \text { falls } \operatorname{tr} A<0 \text { und } c<0 \text { ist, } \\
0 \text { sonst. }
\end{array}\right. \\
& \delta(A)=\left\{\begin{array}{l}
1, \text { falls } \operatorname{tr} A<0 \text { oder falls } \operatorname{tr} A=0 \text { und } c>0 \text { ist, } \\
0 \text { sonst. }
\end{array}\right. \\
& \varepsilon(A)=\left\{\begin{array}{l}
\operatorname{sgn} c, \text { falls } c \neq 0, \\
-\operatorname{sgn} d, \text { falls } c=0 \text { ist. }
\end{array}\right.
\end{aligned}
$$

Für sie gilt offenbar

$$
2 \beta(A)=\delta(A)(1-\varepsilon(A)),
$$

und $\delta$ ist eine Klassenfunktion auf $S L_{2}(\mathbf{R})$, d.h. konstant auf den Klassen konjugierter Matrizen. Ein Zweig des Logarithmus von $j(A, z)$ bzw. von $J(A, z)$ wird festgelegt durch

$$
\left\{\begin{array}{l}
l(A, z):=l_{1}(A, z)+2 \pi i \beta(A), \\
L(A, z):=-2 l_{1}\left((-1)^{\delta(A)} A, z\right) .
\end{array}\right.
$$

Es ist $l_{1}(A, z)-l_{1}(-A, z)=\pi i \varepsilon(A), A \in S L_{2}(\mathbf{R})$, also liefert (2.3)

$$
L(A, z)=-2 l_{1}(A, z)+2 \pi i \delta(A) \varepsilon(A)=-2 l(A, z)+2 \pi i \delta(A) .
$$

Entsprechend gilt für die zu $l$ und $L$ nach (2.1) gehörigen 2-Kozyklen $w$ und $W$

$$
\left\{\begin{array}{l}
\text { (a) } w(A, B)=w_{1}(A, B)+\beta(A B)-\beta(A)-\beta(B), \\
\text { (b) } W(A, B)=-2 w_{1}(A, B)+\delta(A B) \varepsilon(A B)-\delta(A) \varepsilon(A)-\delta(B) \varepsilon(B) \\
\text { (c) } W(A, B)=-2 w(A, B)+\delta(A B)-\delta(A)-\delta(B) .
\end{array}\right.
$$

Wir zeigen jetzt

Lemma 2. Für alle $A_{1}, A_{2} \in S L_{2}(\mathbf{R})$ ist $W\left(A_{1}, A_{2}\right) \in\{-1,0,1\}$. 
Beweis. Sei $A_{0}=A_{1} A_{2}, A_{k}=\left(\begin{array}{ll}a_{k} & b_{k} \\ c_{k} & d_{k}\end{array}\right), k=0,1,2$. Aus der Definition (2.4) hat man für $A \in S L_{2}(\mathbf{R}), z \in \mathfrak{S}:$

$$
L(A, z)=L(-A, z), \quad|\operatorname{Im} L(A, z)|<2 \pi
$$

und, falls $A \infty=\infty$, sogar $\operatorname{Im} L(A, z)=0$. Deshalb ist stets $\left|W\left(A_{1}, A_{2}\right)\right| \leqq 2$, und $\left|W\left(A_{1}, A_{2}\right)\right| \leqq 1$, falls $A_{k} \infty=\infty$ für ein $k$ ist. Wegen (2.6) (b) genügt also zu zeigen: Ist $\delta\left(A_{1}\right)=\delta\left(A_{2}\right)=0, c_{0} c_{1} c_{2} \neq 0$ und $w_{1}\left(A_{1}, A_{2}\right) \neq 0$, so ist $\operatorname{tr}\left(A_{1} A_{2}\right)<0$. Nun bedeutet hier $w_{1}\left(A_{1}, A_{2}\right) \neq 0$ wegen (2.2), daß $c_{0} c_{1}<0$ und $c_{1} c_{2}>0$ ist. Dann haben wir

$$
\frac{c_{0}}{c_{2}}=\frac{c_{1}}{c_{2}} a_{2}+d_{1}<0 \text { und } a_{1}+\frac{c_{1}}{c_{2}} d_{2}=\left(a_{1}+d_{1}\right)+\frac{c_{1}}{c_{2}}\left(a_{2}+d_{2}\right)-\frac{c_{0}}{c_{2}}>0 \text {. }
$$

Daraus folgt

$$
\operatorname{tr} A_{0}=a_{1} a_{2}+b_{1} c_{2}+c_{1} b_{2}+d_{1} d_{2}=\frac{c_{2}}{c_{1}}\left(\frac{c_{1}}{c_{2}} a_{2}+d_{1}\right)\left(a_{1}+\frac{c_{1}}{c_{2}} d_{2}\right)-\left(\frac{c_{1}}{c_{2}}+\frac{c_{2}}{c_{1}}\right)<0 .
$$

Lemma 2 zusammen mit (2.6) (c) ergibt für alle $A_{1}, A_{2}:-3 \leqq 2 w\left(A_{1}, A_{2}\right) \leqq 2$; wegen $w\left(A_{1}, A_{2}\right) \in \mathbf{Z}$ haben wir also

Korollar 1. Für alle $A_{1}, A_{2} \in S L_{2}(\mathbf{R})$ ist $w\left(A_{1}, A_{2}\right) \in\{-1,0,1\}$.

KoRollar 2. $W(A, B)=0$ gilt genau dann, wenn $\delta(A)+\delta(B) \equiv \delta(A B)(\bmod 2)$ ist.

Als nächstes beweisen wir

$$
\left\{\begin{array}{l}
\text { (a) } W\left(A, A^{-1}\right)=0, \text { falls } \operatorname{tr} A \neq 0 . \\
\text { (b) } w\left(A, A^{-1}\right)=0 \text { oder }-1 \text {, jenachdem } \operatorname{tr} A \geqq 0 \text { oder } \operatorname{tr} A<0 \text { ist. } \\
\text { (c) } W(A, B)=-1 \text {, falls } \operatorname{tr} A=\operatorname{tr} B=0 .
\end{array}\right.
$$

Aussage (a) ist eine unmittelbare Folgerung aus Korollar 2. Mit (2.6) (c) ergibt sich aus ihr die Aussage (b) für den Fall tr $A \neq 0$. Im Fall tr $A=0$ ist $w\left(A, A^{-1}\right)=w_{1}\left(A, A^{-1}\right)=0$ und $\delta\left(A A^{-1}\right)-\delta(A)-\delta\left(A^{-1}\right)=-1$, und (2.6) (c) zeigt $W\left(A, A^{-1}\right)=-1$. Bleibt also Aussage (c) für den Fall $B \neq A^{-1}$. Dann ist nach (1.2) (c) zusammen mit dem Bewiesenen

$$
W(A, B)+W\left(B^{-1}, A^{-1}\right)=-W\left(A B, B^{-1} A^{-1}\right)+W\left(A, A^{-1}\right)+W\left(B, B^{-1}\right)=-2,
$$

weil $\operatorname{tr}(A B) \neq 0$ ist. Die Behauptung folgt damit aus Lemma 2.

Lemma 3. Für alle $A, B \in S L_{2}(\mathbf{R})$ ist $W(A, B)=W(B, A)$ und $w(A, B)=w(B, A)$.

Beweis. Aus (2.6) (c) kommt $W(A, B)-W(B, A)=-2(w(A, B)-w(B, A))$. Die Matrizen $T=\left(\begin{array}{rr}0 & 1 \\ -1 & 0\end{array}\right), U^{r}=\left(\begin{array}{ll}1 & r \\ 0 & 1\end{array}\right), r \in \mathbf{R}$, bilden ein Erzeugendensystem von $S L_{2}(\mathbf{R})$ als Halbgruppe. Wegen (1.4) genügt deshalb, für alle $A \in S L_{2}(\mathbf{R})$ zu zeigen

$$
\begin{gathered}
\left|W\left(A, U^{r}\right)-W\left(U^{r}, A\right)\right|<2, \\
|W(A, T)-W(T, A)|<2 .
\end{gathered}
$$


Aus der Definition von $W$ hat man nun, da $L\left(U^{r}, z\right)=0$ ist,

$$
2 \pi i\left(W\left(A, U^{r}\right)-W\left(U^{r}, A\right)\right)=L\left(A U^{r}, U^{-r} z\right)-L\left(U^{r} A, z\right) .
$$

Weil stets $|\operatorname{Im} L(X, z)|<2 \pi$ ist, folgt Aussage (1). Wegen

$$
W(A, T)-W(T, A)=W(T A, T)-W(T, A T)
$$

ist nach (2.7) (c) die Aussage (2) jedenfalls richtig, wenn $\operatorname{tr} A=0 \operatorname{oder} \operatorname{tr}(A T)=0$ ist. Nun ist $\operatorname{tr}(A T)=\operatorname{tr}\left(T^{-1} A^{-1}\right)$, und (1.2), (2.7) liefern

$$
W(T, A)-W(A, T)=W\left(T^{-1}, A^{-1}\right)-W\left(A^{-1}, T^{-1}\right) .
$$

Im Falle $\operatorname{tr} A \neq 0, \operatorname{tr}(A T) \neq 0$ ist ferner

$$
\delta(A)+\delta(T) \equiv \delta(A T)(\bmod 2) \operatorname{oder} \delta\left(T^{-1}\right)+\delta\left(A^{-1}\right) \equiv \delta\left(T^{-1} A^{-1}\right)(\bmod 2) .
$$

Also ist nach Lemma 2 und Korollar 2 die Aussage (2) auch in diesem Falle richtig.

$$
\begin{aligned}
& \text { Wir setzen für } A=\left(\begin{array}{ll}
a & b \\
c & d
\end{array}\right) \in S L_{2}(\mathbf{R}) \\
& \qquad A^{*}:=\left(\begin{array}{rr}
a & -b \\
-c & d
\end{array}\right)=\left(\begin{array}{rr}
-1 & 0 \\
0 & 1
\end{array}\right) A\left(\begin{array}{rr}
-1 & 0 \\
0 & 1
\end{array}\right) .
\end{aligned}
$$

(2.8) Für alle $A \in S L_{2}(\mathbf{R}), z \in \mathfrak{Y}$ gilt

$$
L\left(A^{*},-\bar{z}\right)-\overline{L(A, z)}=-2 \pi i W\left(A, A^{-1}\right) \text { und } l\left(A^{*},-\bar{z}\right)-\overline{l(A, z)}=-2 \pi i w\left(A, A^{-1}\right)
$$

Beweis. Im Falle $\operatorname{tr} A \geqq 0$ ist $l(A, z)=l_{1}(A, z)=\overline{l_{1}\left(A^{*},-\bar{z}\right)}$. Also stimmt dann die zweite Behauptung. Aus (2.5) kommt ferner

$$
L\left(A^{*},-\bar{z}\right)-\overline{L(A, z)}=-2\left(l\left(A^{*},-\bar{z}\right)-\overline{l(A, z)}\right)+2 \pi i\left(\delta(A)+\delta\left(A^{*}\right)\right)
$$

Da stets $L(A, z)=L(-A, z)$ und $(-A)^{*}=-A^{*}$ ist, folgt mit (2.7) also die erste Behauptung. Und aus ihr gewinnt man unter nochmaliger Anwendung von (2.7) auch die zweite Behauptung für den Fall tr $A<0$.

Sei für $x \in \operatorname{Rd} \mathfrak{S}=\mathbf{R} \cup\{\infty\} G_{x}$ die Untergruppe der $A \in S L_{2}(\mathbf{R})$ mit $A x=x$. Sind $A, B \in G_{\infty}$, so ist $\delta(A)+\delta(B) \equiv \delta(A B)(\bmod 2)$, also nach Korollar 2 zu Lemma $2 W(A, B)=0$. Unter Berücksichtigung von (1.3) hat man also

(2.9) Besitzen $A, B \in S L_{2}(\mathbf{R})$ einen gemeinsamen Fixpunkt auf $\operatorname{Rd} \mathfrak{G}$, so ist $W(A, B)=0$.

(2.10) Es sei $n>1$ eine natürliche Zahl und

$$
A=\left(\begin{array}{rr}
\cos \frac{\pi}{n} & \sin \frac{\pi}{n} \\
-\sin \frac{\pi}{n} & \cos \frac{\pi}{n}
\end{array}\right)
$$

Dann gilt $\sum_{k=1}^{n-1} W\left(A^{k}, A\right)=-1$. 
Beweis. Für $n=2$ ist die Behauptung in (2.7) (c) bewiesen. Sei also $n>2$. Es ist

$$
A^{k}=\left(\begin{array}{cc}
\cos \frac{\pi k}{n} & \sin \frac{\pi k}{n} \\
-\sin \frac{\pi k}{n} & \cos \frac{\pi k}{n}
\end{array}\right)
$$

und $\delta\left(A^{k}\right)=0$ für $1 \leqq k \leqq \frac{1}{2} n, \delta\left(A^{k}\right)=1$ für $\frac{1}{2} n<k \leqq n$. Nach Korollar 2 zu Lemma 2 besitzt die Summe also genau einen von 0 verschiedenen Summanden, nämlich für $k \leqq \frac{1}{2} n<k+1$. Dann ist aber $w_{1}\left(A^{k}, A\right)=0$, also nach (2.6) (b) $W\left(A^{k}, A\right)=-\delta\left(A^{k+1}\right)=-1$.

3. Sei $\Gamma$ eine diskrete Untergruppe von $S L_{2}(\mathbf{R})$. Als automorphe Form zu $\Gamma$ bezeichnen wir jede in $\mathfrak{g}$ meromorphe Funktion $f \neq 0$, welche einer Identität

$$
f(A z) J(A, z)^{g}=\chi(A) f(z), \quad A \in \bar{\Gamma},
$$

genügt. Darin sind das Gewicht $g$ und der Multiplikator $\chi(A)$ von $f$ komplexe Zahlen, und es ist $J(A, z)^{g}=\exp (g L(A, z))$. Wegen $\chi(A B)=\chi(A) \chi(B) \exp (2 \pi i g W(A, B))$ ist $\chi$ eine Klassenfunktion auf $\Gamma$. Die Funktion $f=0$ sei automorphe Form zu jeder Gruppe $\Gamma$, zu jedem Gewicht und zu jedem Multiplikator $\chi$. Den C-Modul aller automorphen Formen zu $\Gamma, g, \chi$ bezeichnen wir mit $\mathscr{C}(\Gamma, g, \chi)$. Durch $f \rightarrow f^{C}, f^{C}(z):=f(C z) J(C, z)^{g}$ hat man für jedes $C \in S L_{2}(\mathbf{R})$ einen Isomorphismus von $\mathscr{C}(\Gamma, g, \chi)$ auf $\mathscr{C}\left(C^{-1} \Gamma C, g, \chi_{c}\right)$, wo $\chi_{c}\left(C^{-1} A C\right)=\chi(A)$ für alle $A \in \Gamma$ ist. Die Abbildung $f \rightarrow f^{*}, f^{*}(z)=\overline{f(-\bar{z})}$ liefert, wie man an (2.8) sieht, einen Isomorphismus von $\mathscr{C}(\Gamma, g, \chi)$ auf $\mathscr{C}\left(\Gamma^{*}, \bar{g}, \chi^{*}\right)$, wo $\Gamma^{*}=\left(\begin{array}{rr}-1 & 0 \\ 0 & 1\end{array}\right) \Gamma\left(\begin{array}{rr}-1 & 0 \\ 0 & 1\end{array}\right)$ und $\left.\chi^{*}\left(A^{*}\right)=\overline{\chi\left(A^{-1}\right.}\right)^{-1}$ ist.

Die nullstellenfreien holomorphen automorphen Formen zu $\Gamma$ bilden unter der Multiplikation eine Gruppe $\mathscr{E}(\Gamma)$. Für jedes $f \in \mathscr{E}(\Gamma) \cap \mathscr{C}(\Gamma, g, \chi)$ ist

$$
2 \pi i \omega(f, A):=\int_{z}^{A z} \frac{f^{\prime}(t)}{f(t)} d t+g L(A, z), \quad A \in \Gamma,
$$

unabhängig von $z$. Offenbar ist $\chi(A)=\exp (2 \pi i \omega(f, A))$. Es gelten für alle $A, B \in \Gamma$; $f, f_{1}, f_{2} \in \mathscr{E}(\Gamma) ; C \in S L_{2}(\mathbf{R})$ die Formeln
(a) $\omega(c, A)=0$, falls $c \in \mathbf{C}-\{0\}$
(b) $\omega\left(f_{1} f_{2}, A\right)=\omega\left(f_{1}, A\right)+\omega\left(f_{2}, A\right)$
(c) $\omega(f, A B)=\omega(f, A)+\omega(f, B)+g W(A, B)$
(d) $\omega\left(f^{C}, C^{-1} A C\right)=\omega(f, A)$
(e) $\left.\omega\left(f^{*}, A^{*}\right)=\overline{\omega\left(f, A^{-1}\right.}\right)$.

(a), (b) und (c) sind direkt an der Definition von $\omega$ abzulesen. Um (d) zu zeigen, setzen wir $B=C^{-1} A C$ und erhalten 


$$
\begin{aligned}
2 \pi i \omega(f, A) & =\int_{C z}^{C B z} \frac{f^{\prime}(t)}{f(t)} d t+g L(A, C z) \\
& =\int_{z}^{B z} \frac{f^{\prime}(C x)}{f(C x)} J(C, x) d x+g L(A, C z) \text { nach der Substitution } t=C x \\
& =\int_{z}^{B z} \frac{\left(f^{C}\right)^{\prime}(x)}{f^{C}(x)} d x-g \int_{z}^{B z} \frac{J^{\prime}(C x)}{J(C x)} d x+g L(A, C z) \text { nach Definition von } f^{C} \\
& =2 \pi i \omega\left(f^{C}, B\right)+2 \pi i g\left(W\left(C, C^{-1} A C\right)-W(A, C)\right) \text { nach Definition von } \omega \text { und } W \\
& =2 \pi i \omega\left(f^{C}, C^{-1} A C\right) \text { wegen Lemma } 3 \text { und }(1.3) .
\end{aligned}
$$

Formel (e) wird so eingesehen: Es ist $\left(f^{*}\right)^{\prime}(z)=-\overline{f^{\prime}(-\bar{z})}$. Sei $t(s), 0 \leqq s \leqq 1$, eine differenzierbare Kurve in $\mathfrak{g}$ mit $t(0)=-\bar{z}, t(1)=-\overline{A z}=A^{*}(-\bar{z})$. Dann ist $t^{*}(s):=-\overline{t(s)}$ die aus $t$ durch Spiegelung an der imaginären Achse entstehende Kurve. Für sie gilt $t^{*}(0)=z$, $t^{*}(1)=A z, \frac{d t^{*}}{d s}=-\overline{\left(\frac{d t}{d s}\right)}$. Damit wird

$$
\begin{aligned}
2 \pi i \omega\left(f^{*}, A^{*}\right) & =\int_{0}^{1} \frac{\left(f^{*}\right)^{\prime}(t(s))}{f^{*}(t(s))} \frac{d t}{d s} d s+\bar{g} L\left(A^{*},-\bar{z}\right) \\
& =\int_{0}^{1} \overline{\frac{f^{\prime}\left(t^{*}(s)\right)}{f\left(t^{*}(s)\right)} \frac{d t^{*}}{d s}} d s+\overline{g L(A, z)}-2 \pi i \bar{g} W\left(A, A^{-1}\right) \text { nach }(2.8) \\
& =2 \pi i \overline{\omega\left(f, A^{-1}\right)} \text { nach Definition von } \omega \text { und (3.3) (c). }
\end{aligned}
$$

Das bekannteste Beispiel liefert die Dedekindsche Funktion $\eta$ für den Fall $\Gamma=S L_{2}(Z)$. Dedekind [1] hat einen expliziten Ausdruck für $\omega(\eta, A)$ berechnet, in dem als wesentlicher Bestandteil die nach ihm benannten Summen auftreten. Über sie existiert eine umfangreiche Literatur (vgl. Rademacher [9], Dieter [3]). Das Hauptinteresse liegt dort auf der arithmetischen Herleitung von Identitäten für die Dedekindschen Summen. Die meisten von ihnen sind Spezialfälle der Formeln (3.3). Man hat nur zu beachten, daß $\eta=\eta^{*}$ ist.

Für diskrete Untergruppen $\Gamma$ von $S L_{2}(\mathbf{R})$ sei

$$
E: 0 \rightarrow \underset{q}{\mathrm{C}} \underset{\mathrm{\Gamma}}{\mathrm{\Gamma}} \underset{\mathrm{p}}{\rightarrow} \Gamma \rightarrow 1
$$

die durch $W$ definierte Gruppenerweiterung. Weil stets $W(A, B)=W(-A, B)=W(A,-B)$ ist, kann $E$ auch als Gruppenerweiterung von $C$ mit $\hat{\Gamma}:=\Gamma\langle-I\rangle\langle-I\rangle$ anstelle von $\Gamma$ aufgefaßt werden. Die Existenz einer Abbildung $\omega: \Gamma \rightarrow C$ mit der Eigenschaft

$$
\omega(A B)=\omega(A)+\omega(B)+W(A, B), \quad A, B \in \Gamma,
$$

bedeutet dasselbe wie die Existenz eines Hcmomorphismus $v: \Gamma \rightarrow \widetilde{\Gamma}$ mit $p \circ v=\operatorname{Id}_{\Gamma}$, also das Zerfallen von $E$ ( $v$ wird dann durch $v(A)=(\omega(A), A)$ gegeben). Ist $\Gamma$ freies Produkt zyklischer Gruppen, so ist $H^{2}(\Gamma, D)=0$ für jede dividierbare abelsche Gruppe $D$, eine unmittelbare 
Konsequenz der Tatsache, da $H^{2}(Z, D)=0$ ist für zyklische Gruppen $Z$ (z.B. Huppert [4], Satz 16.20, p. 118). Dann ist also immer die Existenz einer Abbildung $\omega$ mit der Eigenschaft (3.4) gesichert.-Sind $\omega_{1}, \omega_{2}: \Gamma \rightarrow \mathbf{C}$ zwei solche Abbildungen, so ist $\phi=\omega_{1}-\omega_{2}$ ein Homomorphismus von $\Gamma$ in die additive Gruppe von $\mathbf{C}$, in dessen Kern die Matrix $-I$ liegt. Ist nun $\hat{\Gamma}$ freies Produkt von zyklischen Gruppen endlicher Ordnung, so ist $\phi$ notwendigerweise trivial. Also existiert dann eine und nur eine Abbildung $\omega$ mit der Eigenschaft (3.4).

(3.5) Ist $\Gamma$ freies Produkt von zyklischen Gruppen endlicher Ordnung und ist $\Gamma=$ $\Gamma^{*}=\left(\begin{array}{rr}-1 & 0 \\ 0 & 1\end{array}\right) \Gamma\left(\begin{array}{rr}-1 & 0 \\ 0 & 1\end{array}\right)$, so gilt für die Abbildung $\omega$ mit der Eigenschaft (3.4) die Identität $\omega\left(A^{*}\right)=\omega\left(A^{-1}\right), \quad A \in \Gamma$.

Beweis. Aus (2.8) und (1.2) (c) hat man für alle $A, B \in S L_{2}(\mathbf{R})$

$$
\begin{aligned}
W\left(A^{*}, B^{*}\right) & =\frac{1}{2 \pi i}\left(L\left(A^{*} B^{*},-\bar{z}\right)-L\left(A^{*},-\overline{B(z)}\right)-L\left(B^{*},-\bar{z}\right)\right) \\
& \left.=\frac{1}{2 \pi i} \overline{(L(A B, z)}-\overline{L(A, B z)}-\overline{L(B, z)}\right)+W\left(A, A^{-1}\right)-W\left(A B, B^{-1} A^{-1}\right)+W\left(B, B^{-1}\right) \\
& =W\left(B^{-1}, A^{-1}\right) .
\end{aligned}
$$

Es genügt also, die Behauptung für die Elemente $A$ eines Erzeugendensystems $\mathscr{S}$ von $\Gamma \mathrm{zu}$ zeigen. Nach der Voraussetzung über $\widehat{\Gamma}$ können die $A \in \mathscr{S}$ in der Form

$$
A=B\left(\begin{array}{rr}
\cos \frac{\pi}{n} & \sin \frac{\pi}{n} \\
-\sin \frac{\pi}{n} & \cos \frac{\pi}{n}
\end{array}\right) B^{-1}, \quad B \in S L_{2}(\mathbf{R}), \quad n \in \mathbf{N},
$$

gewählt werden. Dann ist nach (3.4)

$$
0=\omega\left(A^{n}\right)=n \omega(A)+\sum_{k=1}^{n-1} W\left(A^{k}, A\right)
$$

also wegen (2.10) $\omega(A)=1 / n$. Da $A^{*}$ in $S L_{2}(\mathbf{R})$ konjugiert ist zu $A^{-1}$, gilt $\omega\left(A^{*}\right)=\omega\left(A^{-1}\right)$. Nebenbei ergibt sich, daß $\omega(\Gamma)$ in dem von den $\omega(A), A \in \mathscr{S}$, erzeugten $\mathbf{Z}$-Modul liegt.

Wir wenden das auf die rationale Modulgruppe $\Gamma=S L_{2}(\mathrm{Z})$ an. $\hat{\Gamma}$ ist dann freies Produkt einer zyklischen Gruppe der Ordnung 2 mit einer zyklischen Gruppe der Ordnung 3. Also ist $\omega(\Gamma) \subset \frac{1}{b} \mathbf{Z}$.

Lemma 4. Für die Abbildung $\omega: S L_{2}(\mathbf{Z}) \rightarrow \mathbf{C}$ mit $\omega(A B)=\omega(A)+\omega(B)+W(A, B)$ gilt

$$
\omega\left(U^{m}\right)=m / 6 \quad(m \in \mathbf{Z})
$$


und für $A=\left(\begin{array}{ll}a & b \\ c & d\end{array}\right)$, falls $c \neq 0$,

$$
\omega(A)=\frac{a+d}{6 c}+\operatorname{sgn} c\left(\delta(A)-\frac{1}{2}\right)+\sigma(c, d) .
$$

Darin hat $\sigma$ für teilerfremde $c, d \in \mathbf{Z}$ die Eigenschaften

$$
\begin{array}{r}
\sigma(c, d)=\sigma(-c,-d)=-\sigma(-c, d)=\sigma(c, c+d) ; \\
\sigma(c, d)+\sigma(d, c)=\frac{1}{2} \operatorname{sgn} c \operatorname{sgn} d-\frac{c^{2}+d^{2}+1}{6 c d}
\end{array}
$$

falls $c d \neq 0$ ist.

Beweis. Für

$$
T=\left(\begin{array}{rr}
0 & 1 \\
-1 & 0
\end{array}\right), \quad U=\left(\begin{array}{ll}
1 & 1 \\
0 & 1
\end{array}\right), \quad T U^{-1}=\left(\begin{array}{rr}
0 & 1 \\
-1 & 1
\end{array}\right)
$$

ist nach dem Beweis von (3.5) $\omega(T)=\frac{1}{2}$ und $\omega\left(T U^{-1}\right)=\frac{1}{3}$. Aus Korollar 2 zu Lemma 2 folgt $W\left(T, U T^{-1}\right)=0$, also

$$
\omega(U)=\omega\left(T U T^{-1}\right)=\omega(T)+\omega\left(U T^{-1}\right)=\frac{1}{2}-\frac{1}{3} .
$$

Das liefert insbesondere $\omega\left(U^{m}\right)=m / 6$. Für $A=\left(\begin{array}{ll}a & b \\ c & d\end{array}\right)$ mit $c \neq 0$ ist wegen $w_{1}\left(U^{m}, A\right)=0$ nach (2.6) (b)

$$
W\left(U^{m}, A\right)=\operatorname{sgn} c\left(\delta\left(U^{m} A\right)-\delta(A)\right) .
$$

Nun ist $\left\{U^{m} A ; m \in \mathbf{Z}\right\}$ die Menge aller Matrizen aus $S L_{2}(\mathbf{Z})$ mit derselben zweiten Zeile wie A. Also hängt

$$
\sigma(c, d):=\omega(A)-\frac{a+d}{6 c}-\operatorname{sgn} c\left(\delta(A)-\frac{1}{2}\right)
$$

nur von der zweiten Zeile $(c, d)$ von $A$ ab. Wegen $\omega(A)=\omega(-A)$ gilt $\sigma(c, d)=\sigma(-c,-d)$, und $\omega(U A)=\omega(A U)$ liefert $\sigma(c, d)=\sigma(c, c+d)$. Ist $\operatorname{tr} A>0$, so gilt wegen (3.5) und (2.7) (a)

$$
0=\omega(A)+\omega\left(A^{*}\right)=\sigma(c, d)+\sigma(-c, d) .
$$

Schließlich braucht die letzte Aussage nur für $c>0, d>0$ gezeigt zu werden. Man wählt dazu $A$ so, daß $a+d>0$ und $b-c>0$ ist. Es gilt $A=B T$ mit $B=\left(\begin{array}{ll}b & -a \\ d & -c\end{array}\right)$, also wegen Korollar 2 zu Lemma $2 \omega(A)=\omega(B)+\frac{1}{2} ;$ und das ergibt mit dem Vorangehenden

$$
\sigma(c, d)+\sigma(d, c)=\frac{b-c}{6 d}-\frac{a+d}{6 c}+\frac{1}{2}=\frac{1}{2}-\frac{c^{2}+d^{2}+1}{6 c d} .
$$


Wir greifen den Fall tr $A=2$ heraus. Für $A=\left(\begin{array}{ll}a & b \\ c & d\end{array}\right), B=\left(\begin{array}{ll}\alpha & \beta \\ \gamma & \delta\end{array}\right) \in S L_{2}(\mathbf{Z})$ setzt man

$$
v(A)=\left(\begin{array}{c}
-b \\
a-d \\
c
\end{array}\right), \quad D(B)=\left(\begin{array}{ccc}
\alpha^{2} & \alpha \beta & \beta^{2} \\
2 \alpha \gamma & \alpha \delta+\beta \gamma & 2 \beta \delta \\
\gamma^{2} & \gamma \delta & \delta^{2}
\end{array}\right)
$$

und hat $v\left(B A B^{-1}\right)=D(B) v(A)$. Es folgt daraus, daß der (positive) größte gemeinsame Teiler ggT $(a-d, b, c)$ eine Klassenfunktion auf $S L_{2}(Z)$ ist, und im Falle $\operatorname{tr} A=2$ auch $\operatorname{sgn}(b-c)$. Aus $\omega\left(U^{m}\right)=m / 6$ für $m \in \mathbf{Z}$ gewinnt man deshalb

(3.6) Für alle $A=\left(\begin{array}{ll}a & b \\ c & d\end{array}\right) \in S L_{2}(\mathbf{Z})$ mit $\operatorname{tr} A=2$ gilt $\omega(A)=\frac{1}{6} \operatorname{sgn}(b-c) \operatorname{ggT}(a-d, b, c)$. Dedekind [1] erhält für $A=\left(\begin{array}{ll}a & b \\ c & d\end{array}\right) \in S L_{2}(Z)$ mit positivem $c$

$$
\log \eta(A z)-\log \eta(z)=\frac{1}{2} \log \frac{c z+d}{i}+\pi i\left\{\frac{a+d}{12 c}-s(d, c)\right\} \text {. }
$$

Darin ist der Logarithmus durch $-\pi / 2<\operatorname{Im} \log x<\pi / 2$ in $\operatorname{Re} x>0$ normiert, $s(d, c)$ ist die sog. Dedekindsche Summe

$$
\begin{aligned}
& s^{\prime}(d, c)=\sum_{m(\bmod c)} B_{1}\left(\frac{m}{c}\right) B_{1}\left(\frac{m d}{c}\right) \quad \text { und } \\
& B_{1}(x)=\left\{\begin{array}{lll}
x-[x]-\frac{1}{2}, & \text { falls } & x \in \mathbf{R}-\mathbf{Z} \\
0, & \text { falls } & x \in \mathbf{Z}
\end{array}\right.
\end{aligned}
$$

bezeichnet die durch das erste Bernoulli-Polynom definierte periodische Funktion. $s(d, c)$ hat für teilerfremde $c, d \in \mathbf{Z}, c>0$, die beiden trivialen Eigenschaften $s(d, c)=-s(-d, c)=$ $s(d+c, c)$, und erfüllt das Reziprozitätsgesetz

$$
s(d, c)+s(c, d)=-\frac{1}{4}+\frac{c^{2}+d^{2}+1}{12 c d} \quad(c>0, d>0) .
$$

$s$ ist dadurch eindeutig bestimmt. Insbesondere haben wir

$$
\sigma(c, d)=-2 s(d, c), \text { falls } c>0, d>0 .
$$

Es ist bemerkenswert, daß diese Identität ohne Benutzung der Dedekindschen Funktion bewiesen werden kann, weil elementar-arithmetische Beweise für das Reziprozitätsgesetz existieren (s. z.B. Mordell [6]).

4. In diesem Abschnitt sei stets $\Gamma=S L_{2}(Z)$, für natürliche Zahlen $n \quad \Gamma(n)$ die Hauptkongruenzgruppe der Stufe $n$, d.i. die Untergruppe $\operatorname{der} A \equiv I(\bmod n)$ von $\Gamma$, ferner $\Delta(n)$ der von $U^{n}$ erzeugte Normalteiler von $\Gamma$. Wohlfahrt [10] hat mit Hilfe der Dedekindschen Summen eine Serie von Nichtkongruenzuntergruppen von $\Gamma$ angegeben, die hier näher untersucht werden. Dazu ist der folgende Satz von zentraler Bedeutung. 
SATZ 1. (Fricke-Wohlfahrt) Ist $t$ ein positiver Teiler der natürlichen Zahl $m$, so gilt

$$
\Gamma(m) \Delta(t)=\Gamma(t) .
$$

Der Vollständigkeit halber gebe ich noch einen Beweis, in Anlehnung an Wohlfahrt [11]. Es muß gezeigt werden, da $\beta$ zu jedem $A \in \Gamma(t)$ ein $B \in \Delta(t) \operatorname{mit} A \equiv B(\bmod m)$ existiert. Ist $A=\left(\begin{array}{ll}a & b \\ c & d\end{array}\right)$ und $c \neq \equiv 0(\bmod m)$, so existieren $x, y \in \mathbf{Z}$ mit $c x+d y=1$ und $\operatorname{ggT}(y, m / t)=1$. Man wähle dazu ein $g \in \mathbf{Z}$ mit $y g t \equiv c(\bmod m)$. Dann ist

$$
C=\left(\begin{array}{cc}
1-x y g t & x^{2} g t \\
-y^{2} g t & 1+x y g t
\end{array}\right) \quad \text { in } \quad \Delta(t) \quad \text { und } A C \equiv\left(\begin{array}{ll}
* & * \\
0 & *
\end{array}\right)(\bmod m) \text {. }
$$

Ist $A=\left(\begin{array}{ll}a & b \\ c & d\end{array}\right)$ und $c \equiv 0(\bmod m)$, so gilt $a d-1 \equiv 0(\bmod m)$, also

$$
\left(\begin{array}{cc}
1 & 0 \\
1-d & 1
\end{array}\right)\left(\begin{array}{cc}
a & a-1 \\
1-a & 2-a
\end{array}\right)\left(\begin{array}{cc}
1 & (b+1) d-1 \\
0 & 1
\end{array}\right) \equiv\left(\begin{array}{cc}
1 & 0 \\
1-d & 1
\end{array}\right)\left(\begin{array}{cc}
a & b \\
1-a & d+b(d-1)
\end{array}\right)
$$

$\equiv A(\bmod m)$, und die drei Matrizen auf der linken Seite liegen in $\Delta(t)$. Das beweist den Satz 1 .

Für Untergruppen $\Delta$ von endlichem Index in $\Gamma$ wird nach Wohlfahrt [11] die Stufe von $\Delta$ definiert als kleinste natürliche Zahl $t$ mit $\Delta(t) \subset \Delta . \Delta$ heißt eine Kongruenzuntergruppe von $\Gamma$, wenn für ein $m \in \mathbf{N}$ gilt $\Gamma(m) \subset \Delta$. Nach Satz 1 ist $\Delta$ Kongruenzuntergruppe genau dann, wenn $\Gamma(t) \subset \Delta$ ist.

Mit der Abbildung $\omega$ von Lemma 4 sei $\Omega=6 \omega$. Dann ist $\Omega(\Gamma) \subset Z$. Für natürliche Zahlen $n>1$ sei $\Gamma_{0}(n)$ die Untergruppe $\operatorname{der} A \equiv\left(\begin{array}{ll}* & * \\ 0 & *\end{array}\right)(\bmod n)$ von $\Gamma, N=\left(\begin{array}{ll}n & 0 \\ 0 & 1\end{array}\right)$ und

$$
\Omega_{n}(A):=\Omega\left(N A N^{-1}\right)-\Omega(A), A \in \Gamma_{0}(n) .
$$

Wegen (1.3) ist $\Omega_{n}: \Gamma_{0}(n) \rightarrow Z$ ein Homomorphismus.

(4.1) Ist $A=\left(\begin{array}{ll}a & b \\ c & d\end{array}\right) \in \Gamma_{0}(n)$ und $c>0, d>0$, so gilt

mit

$$
\Omega_{n}(A)=\frac{n-1}{d}\left(b-\frac{c}{n}\left(4 d^{2}-6 d+1\right)\right)+\frac{12}{d} L_{n}(c, d)
$$

Beweis. Wegen Lemma 4 gilt

$$
L_{n}(c, d)=\sum_{m=1}^{d-1} m\left(\left[\frac{m c}{d}\right]-\left[\frac{m c}{n d}\right]\right)
$$

$$
\Omega_{n}(A)=\frac{(a+d)(n-1)}{c}+6 \sigma\left(\frac{c}{n}, d\right)-6 \sigma(c, d)=\frac{n-1}{d}\left(b+\frac{c}{n}\right)+6 \sigma(d, c)-6 \sigma\left(d, \frac{c}{n}\right) .
$$


Nach (3.7) wird unter Verwendung der Identität

$$
\begin{gathered}
\sum_{m=1}^{d-1} B_{1}\left(\frac{m c}{d}\right)=\sum_{m=1}^{d-1} B_{1}\left(\frac{m c}{n d}\right)=0 \\
\sigma(d, c)-\sigma\left(d, \frac{c}{n}\right)=2 s\left(\frac{c}{n}, d\right)-2 s(c, d) \\
=\frac{2}{d} \sum_{m=1}^{d-1} m\left(\frac{m c}{n d}-\frac{m c}{d}\right)+\frac{2}{d} \sum_{m=1}^{d-1} m\left(\left[\frac{c m}{d}\right]-\left[\frac{m c}{n d}\right]\right) \\
=\frac{2 c}{n d}(1-n) \frac{(d-1)(2 d-1)}{6}+\frac{2}{d} L_{n}(c, d) .
\end{gathered}
$$

Daraus folgt die Behauptung.

(4.2) Sind $c, d \in \mathbf{N}$ koprim, $c \equiv 0(\bmod n), d \equiv 1(\bmod 2)$, so gilt mit dem LegendreSymbol $\left(\frac{n}{d}\right)$

$$
(-1)^{L_{n}(c, d)}=\left(\frac{n}{d}\right)(-1)^{c(d-1)(n-1) / 2 n} .
$$

Zum Beweis wird ein Schluß von Rademacher [8] verwendet. Es ist

$$
L_{n}(c, d) \equiv \sum_{m=1}^{d-1}\left(\left[\frac{m c}{d}\right]-\left[\frac{m c}{n d}\right]\right)+\sum_{m=1}^{\frac{1}{2}(d-1)}\left(\left[\frac{2 m c}{d}\right]-\left[\frac{2 m c}{n d}\right]\right)(\bmod 2) .
$$

Durch Abzählen der Gitterpunkte im Inneren der Dreiecke mit den Ecken $(0,0),(d, 0),(d, c)$ bzw. $(0,0),(d, 0),(d, c / n)$ erhält man für die linke Summe $\frac{1}{2}(d-1)(c / n)(n-1)$. Nach Scherings Verallgemeinerung des Gaußschen Lemmas gilt für teilerfremde $c, d \in \mathbf{Z}$ mit positivem $d \equiv 1(\bmod 2)$ für

$$
R=\sum_{m=1}^{\frac{1}{2}(d-1)}\left[\frac{2 m c}{d}\right]:(-1)^{R}=\left(\frac{c}{d}\right)
$$

als $\mathrm{J}$ ist die rechte Summe $\equiv 0$ oder $1(\bmod 2)$ jenachdem $\left(\frac{c}{d}\right)\left(\frac{c / n}{d}\right)=\left(\frac{n}{d}\right)$ gleich 1 oder -1 ist.

(4.3) Ist $n \equiv 1(\bmod 24)$, so gilt für alle $A=\left(\begin{array}{ll}a & b \\ c & d\end{array}\right) \in \Gamma_{0}(n) \quad \exp \left[2 \pi i \Omega_{n}(A) / 24\right]=\left(\frac{d}{n}\right)$.

Zum Beweis darf wegen $\Omega_{n}(A)=\Omega_{n}(-A)=-\Omega_{n}\left(A^{*}\right) \quad c>0$ und $d>0$ vorausgesetzt werden. Es ist

$$
\begin{gathered}
\Omega_{n}\left(\begin{array}{ll}
1 & 1 \\
0 & 1
\end{array}\right)=n-1 \equiv 0 \quad(\bmod 24) \\
\left(\begin{array}{ll}
a & b \\
c & d
\end{array}\right)\left(\begin{array}{ll}
1 & 1 \\
0 & 1
\end{array}\right)=\left(\begin{array}{ll}
a & a+b \\
c & c+d
\end{array}\right) \text { und }\left(\frac{d}{n}\right)=\left(\frac{c+d}{n}\right) .
\end{gathered}
$$


Weil $d$ oder $c+d$ ungerade ist, darf weiterhin angenommen werden, daß $d$ ungerade ist. Dann gilt wegen (4.1), (4.2) nach dem quadratischen Reziprozitätsgesetz

$$
\exp \left(\frac{2 \pi i \Omega_{n}(A)}{24}\right)=\left(\frac{n}{d}\right)=\left(\frac{d}{n}\right) \text {. }
$$

Aussage (4.3) wird für zu 6 prime Quadratzahlen $n$ in der Theorie der komplexen Multiplikation zum Nachweis des Hauptidealsatzes der Klassenkörpertheorie benötigt (vgl. Deuring [2]).

Wir definieren die natürlichen Zahlen $\gamma_{n}, \delta_{n}$ durch $\Omega_{n}(\Gamma(n))=\gamma_{n} \mathbf{Z}, \Omega_{n}(\Delta(n))=\delta_{n} \mathbf{Z} . \delta_{n}$ kann sofort explizit angegeben werden: Nach Definition wird $\Delta(n)$ erzeugt durch die Matrizen

$$
A=\left(\begin{array}{cc}
1+x y n & -x^{2} n \\
y^{2} n & 1-x y n
\end{array}\right)
$$

mit teilerfremden $x, y \in \mathbf{Z}$. Wegen (3.6) ist $\Omega_{n}(A)=n-t^{2}, t=\operatorname{ggT}(n, y)$. Das liefert

$$
\delta_{n}=\operatorname{ggT}\left(n-t^{2} ; t \mid n\right) \text {. }
$$

Im nächsten Satz wird $\gamma_{n}$ berechnet und ein Überblick über die sämtlichen Kongruenzuntergruppen unter den Gruppen $\Omega_{n}^{-1}(m \mathbf{Z}), m \in \mathbf{N}$, gegeben.

SATZ 2. Für jede natürliche Zahl $n>1$ gilt

$$
\gamma_{n}=\operatorname{ggT}(n-1,24) \text { und } \bigcap_{m \in N} \Omega_{n}(\Gamma(m n))=24 Z
$$

Beweis. Es sei $\alpha_{2}=4, \alpha_{3}=2$ und $\alpha_{p}=1$ für Primzahlen $p>3$; ferner $\delta_{n}=\prod_{p} p^{\beta_{p}}$. Wegen $\Omega_{n}(\Delta(m n))=m \delta_{n} Z$ ist die Stufe von $\Omega_{n}^{-1}\left(p^{\alpha} Z\right)$ gleich $n$, falls $\alpha \leqq \beta_{p}$, gleich $p^{\alpha-\beta_{p}} n$, falls $\alpha \geqq \beta_{p}$ ist. Ist $\Omega_{n}^{-1}(m Z)$ eine Kongruenzuntergruppe von $\Gamma$, so ist für jede Primzahlpotenz $p^{\alpha}$, welche $m$ teilt, auch $\Omega_{n}^{-1}\left(p^{\alpha} Z\right)$ eine Kongruenzuntergruppe. Nach (4.1) und (4.2) ist sicher $\Omega_{n}(\Gamma(24 n)) \subset 24 Z$. Es ist also neben der ersten Behauptung von Satz 2 zu zeigen, daß für jede Primzahl $p \quad \Omega_{n}^{-1}\left(p^{\alpha_{p}} Z\right)$ eine Nichtkongruenzuntergruppe von $\Gamma$ ist. Wir beginnen mit

(1) Ist $\delta_{n} \neq \equiv 0(\bmod p)$ und $\alpha \in \mathbf{N}$, so existiert eine Matrix

$$
A \in \Gamma\left(p^{\alpha} n\right) \cap \Delta(n) \text { mit } \Omega_{n}(A) \not \equiv 0\left(\bmod p^{\alpha_{p}}\right) .
$$

Daraus folgt mit Satz 1 insbesondere, daß für $\beta_{p}=0 \quad \Omega_{n}^{-1}\left(p^{\alpha_{p}} \mathbf{Z}\right)$ eine Nichtkongruenzuntergruppe ist. Zum Beweis setzen wir $A$ an in der Form

$$
A=\left(\begin{array}{cc}
1 & 0 \\
y^{2} n & 1
\end{array}\right)\left(\begin{array}{cc}
1-x y n & x^{2} n \\
-y^{2} n & 1+x y n
\end{array}\right)=\left(\begin{array}{cc}
1-x y n & x^{2} n \\
-x y^{3} n & 1+x y n+x^{2} y^{2} n^{2}
\end{array}\right)
$$

mit teilerfremden $x, y \in \mathbf{Z}, x \equiv 0\left(\bmod p^{\alpha}\right)$. Dann ist $A \in \Gamma\left(p^{\alpha} n\right)$ und wegen (3.6) gilt

$$
\Omega_{n}(A)=y^{2}(n-1)-n+t^{2}, \quad t=\operatorname{ggT}(n, y) .
$$


Ist $p$ kein Teiler von $n-1$, so wählen wir $t=1$ und $y^{2} \not \equiv 1\left(\bmod p^{\alpha p}\right)$. Dann wird $\Omega_{n}(A)=$ $(n-1)\left(y^{2}-1\right) \not \equiv 0\left(\bmod p^{\alpha_{p}}\right)$. Ist $n-1 \equiv 0(\bmod p)$, so existiert wegen $\delta_{n} \not \equiv 0(\bmod p)$ ein Teiler $t$ von $n$, für den $n-t^{2}$, und damit $\Omega_{n}(A)$, prim zu $p$ wird. Das beweist die Behauptung.

(2) Ist $p=2$ oder 3 und $n \equiv 1(\bmod p), n \neq \equiv 1\left(\bmod p^{2}\right)$, so existiert ein $A \in \Gamma\left(p^{\alpha_{p}-1} n\right) \cap \Delta(n)$ $\operatorname{mit} \Omega_{n}(A) \not \equiv 0\left(\bmod p^{\alpha_{\mathfrak{p}}}\right)$.

(Insbesondere ist dann $\beta_{p}=1$ und $\Omega_{n}^{-1}\left(p^{\alpha_{p}} Z\right)$ eine Nichtkongruenzuntergruppe von $\Gamma$.)

Sei

$$
B=\left(\begin{array}{cc}
1 & n^{2} \\
0 & 1
\end{array}\right)\left(\begin{array}{ll}
1 & 0 \\
n & 1
\end{array}\right)=\left(\begin{array}{cc}
1+n^{3} & n^{2} \\
n & 1
\end{array}\right) \text {. }
$$

Dann ist $\Omega_{n}(B)=(n-1)\left(n^{2}+1\right)$; also $\Omega_{n}(B) \not \equiv 0\left(\bmod 2^{3}\right)$, falls $p=2, \Omega_{n}(B) \not \equiv 0\left(\bmod 3^{2}\right)$, falls $p=3$ ist. Ferner gilt $B \equiv\left(\begin{array}{cc}0 & 1 \\ -1 & 1\end{array}\right)(\bmod 8)$ für $n \equiv-1(\bmod 8), B \equiv\left(\begin{array}{ll}4 & 1 \\ 3 & 1\end{array}\right)(\bmod 8)$ für $n \equiv 3(\bmod 8)$. Jedenfalls ist dann $A:=B^{6} \in \Gamma(8 n)$ und $\Omega_{n}(A)=6 \Omega_{n}(B) \not \equiv 0\left(\bmod 2^{4}\right)$. Ist $n \equiv 1(\bmod 3)$, so ist $B \equiv\left(\begin{array}{ll}2 & 1 \\ 1 & 1\end{array}\right)(\bmod 3)$, also $A:=B^{4} \in \Gamma(3 n)$ und $\Omega_{n}(A) \neq 0\left(\bmod 3^{2}\right)$.

Zum Beweis der restlichen Behauptungen berechnen wir zu natürlichen Zahlen $q, r, s, t$, welche $r t \equiv 1(\bmod q)$ und $s t=n-1$ erfüllen, $\Omega_{n}(A)$ für die Matrix

$$
A=\left(\begin{array}{cc}
1 & s\left(\frac{r t-1}{q}+1\right)-n r \\
0 & 1
\end{array}\right)\left(\begin{array}{cc}
n r-s & \frac{r t-1}{q} \\
q n & t
\end{array}\right)\left(\begin{array}{cc}
t & 1 \\
-n & -s
\end{array}\right) .
$$

Man prüft leicht nach, daß $A \in \Gamma((q-1) n)$ ist. Wir zeigen

$$
\text { (3) } \Omega_{\mathrm{n}}(A)=(n-1)\left\{s\left(\frac{r t-1}{q}+1\right)-n r\right\}+s\left(\frac{r t-1}{q}+q\right) \text {. }
$$

Für jedes $X \in S L_{2}(\mathbf{R})$ ist $X^{\prime}=T X^{-1} T^{-1}$ die zu $X$ transponierte Matrix. Deshalb ist mit $C:=\left(\begin{array}{ll}t & -n \\ 1 & -s\end{array}\right)$ wegen $(3.4),(3.5)$

$$
\Omega_{n}\left(C^{\prime}\right)=\Omega\left(C^{*}\right)-\Omega\left(C^{\prime}\right)=\Omega\left(C^{-1}\right)-\Omega\left(C^{-1}\right)=0,
$$

und für

$$
B:=\left(\begin{array}{cc}
n r-s & \frac{r t-1}{q} \\
q_{n} & t
\end{array}\right)
$$

gilt nach (4.1)

wird

$$
\Omega_{n}(B)=\frac{n-1}{t}\left\{\frac{r t-1}{q}-q\left(4 t^{2}-6 t+1\right)\right\}+\frac{12}{t} L_{n}(q n, t) . \text { Wegen }
$$

$$
\left[\frac{m q n}{t}\right]=m q s+\left[\frac{m q}{t}\right]
$$

und daraus ergibt sich die Behauptung. 
Sind nun $q$ und $n-1$ teilerfremd, so wird $\Omega_{n}(A) \equiv s\left(q^{2}-1\right) / q(\bmod (n-1))$. Wählt man zu jedem Primteiler $p$ von $n-1$ für $t$ die maximale $p$-Potenz in $n-1$ und $q^{2} \not \equiv 1\left(\bmod p^{\alpha_{p}}\right)$, so hat man, daß $\gamma_{n}$ ein Teiler von $n-1$ und 24 ist. Andererseits zeigen (4.1) und (4.2), daß für alle $A \in \Gamma(n) \quad \Omega_{n}(A) \equiv 0(\bmod g g T(n-1,24))$ ist. Damit ist $\gamma_{n}=\operatorname{ggT}(n-1,24)$ bewiesen. Zusätzlich hat sich ergeben

(4) Ist $n \equiv 1\left(\bmod p^{\alpha_{p}}\right)$, so existiert eine Matrix $A \in \Gamma(n) \operatorname{mit} \Omega_{n}(A) \not \equiv 0\left(\bmod p^{\alpha_{p}}\right)$. Für $p=2,3$ kann $A$ in $\Gamma(p n)$ gewählt werden.

Wegen (2) ist damit für $\delta_{n} \equiv 0(\bmod p)$ gezeigt, da $\beta \Omega_{n}{ }^{-1}\left(p^{\alpha_{p}} Z\right)$ eine Nichtkongruenzuntergruppe von $\Gamma$ ist, abgesehen vom Fall $p=2, n \equiv 1 \bmod 4, n \neq \equiv 1 \bmod 16$. Es fehlt also noch der Nachweis von

(5) Ist $n \equiv 5(\bmod 8)$, so existiert ein $A \in \Gamma(4 n) \operatorname{mit} \Omega_{n}(A) \not \equiv 0\left(\bmod 2^{4}\right)$. Ist $n \equiv 9(\bmod 16)$, so existiert ein $A \in \Gamma(2 n)$ mit $\Omega_{n}(A) \not \equiv 0\left(\bmod 2^{4}\right)$.

Dazu wähle man in (3) $t=4$ bzw. $8, r t=16, q=5$ bzw. 3 . Dann wird

$$
\Omega_{n}(A)=(n-1)\left(\frac{n-1}{t}\left(\frac{15}{q}+1\right)-n r\right)+8 \frac{n-1}{t} \equiv 8(\bmod 16) .
$$

\title{
LITERATUR
}

1. R. Dedekind, Erläuterungen zu zwei Fragmenten von Riemann, Gesammelte Werke, Bd 1 (Braunschweig, 1930).

2. M. Deuring, Die Klassenkörper der komplexen Multiplikation, Enzyklopädie der Mathematischen Wissenschaften, Bd I2, Heft 10, Teil II (Stuttgart, 1958).

3. U. Dieter, Beziehungen zwischen Dedekindschen Summen, Abh. Math. Sem. Univ. Hamburg 21 (1957), 109-125.

4. B. Huppert, Endliche Gruppen I (Springer Verlag Berlin, Heidelberg, New York, 1967).

5. S. MacLane, Homology (Springer Verlag Berlin, Göttingen, Heidelberg, 1963).

6. L. J. Mordell, The reciprocity formula for Dedekind sums, Amer. J. Math. 73 (1951), 593-601.

7. H. Petersson, Analytische Theorie der Grenzkreisgruppen I, II, III, Math. Ann. 115 (1938), 23-67, 175-204, 518-572.

8. H. Rademacher, Bestimmung einer gewissen Einheitswurzel in der Theorie der Modulfunktionen, J. London Math. Soc. 7 (1932), 14-19.

9. H. Rademacher, Zur Theorie der Dedekindschen Summen, Math. Z. 63 (1955/56), 445-463.

10. K. Wohlfahrt, Uber Dedekindsche Summen und Untergruppen der Modulgruppe, Abh. Math. Sem. Univ. Hamburg 23 (1959), 5-10.

11. K. Wohlfahrt, An extension of F. Klein's level concept, Illinois J. Math. 8 (1964), 529-535.

\author{
II. Mathematisches INSTITUT deR Universität \\ SCHLOBPLATZ 2 \\ 44 MÜNSTER \\ GeRMANY
}

